\title{
A posteriori necessities in one dimension
}

\section{Eric Johannesson ${ }^{1}$}

Published online: 3 December 2019

() The Author(s) 2019

\begin{abstract}
Arguably, the proposition that Mark Twain is Samuel Clemens and the proposition that water is $\mathrm{H} 2 \mathrm{O}$ are both a posteriori. Nevertheless, they both seem to be necessary. Ever since Davies and Humberstone (Philos Stud 38(1):1-31, 1980), it has been known that two-dimensional semantics can account for this fact. But two-dimensionalism isn't the only theory on the market that purports to do so. In this paper, I will look at two alternatives, one by Scott Soames and one by Kathrin Glüer-Pagin and Peter Pagin, and argue that both of them fail. Regarding the former, I argue that the conceptually possible but metaphysically impossible worlds one is required to postulate are hard to conceive of on closer inspection. As for the latter, the proposal doesn't work for certain modal sentences, and I show that it cannot be easily amended.
\end{abstract}

Keywords A priori - A posteriori - Necessity $\cdot$ Proper names $\cdot$ Natural kind predicates $\cdot$ Two-dimensional semantics $\cdot$ Switcher semantics

\section{Introduction}

Consider the sentences

(1) Mark Twain is Samuel Clemens.

and

(2) If something is a quantity of water then it's a quantity of H2O-molecules.

Intuitively, both express a posteriori truths. Nevertheless, the following sentences are generally taken to be true:

(3) Necessarily, Mark Twain is Samuel Clemens.

and

Eric Johannesson

johannesson.eric@gmail.com

1 Department of Philosophy, Stockholm University, 10691 Stockholm, Sweden 
(4) Necessarily, if something is a quantity of water then it's a quantity of H2Omolecules.

Ever since Davies and Humberstone (1980), it has been known that two-dimensional semantics can account for this fact. The trick is to interpret every name $a$ in a model $\mathcal{M}=\langle W, D, I\rangle$ (where $W$ is a set of possible worlds and $D$ is a domain of objects) as a function $I(a): W \rightarrow D$ rather than as abject $I(a) \in D$, and each $n$ place predicate $P$ as a function $I(P): W \times W \rightarrow \mathcal{P}\left(D^{n}\right)$ rather than as a function $I(P): W \rightarrow \mathcal{P}\left(D^{n}\right)$, and to evaluate each expression relative to a pair of worlds rather than just a single world, in the following way:

$$
\begin{array}{ll}
\text { a. } & \llbracket a \rrbracket_{\mathcal{M}, u, w}=I(a)(u) . \\
\text { b. } & \llbracket P \rrbracket_{\mathcal{M}, u, w}=I(P)(u)(w) . \\
\text { c. } & \llbracket P a_{1} \ldots a_{n} \rrbracket_{\mathcal{M}, u, w}=1 \text { iff }\left\langle\llbracket a_{1} \rrbracket_{\mathcal{M}, u, w}, \ldots, \llbracket a_{n} \rrbracket_{\mathcal{M}, u, w}\right\rangle \in \llbracket P \rrbracket_{\mathcal{M}, u, w} . \\
\text { d. } \llbracket \neg \varphi \rrbracket_{\mathcal{M}, u, w}=1 \text { iff } \llbracket \varphi \rrbracket_{\mathcal{M}, u, w}=0 . \\
\text { e. } \llbracket \varphi \wedge \psi \rrbracket_{\mathcal{M}, u, w}=1 \text { iff } \llbracket \varphi \rrbracket_{\mathcal{M}, u, w}=1 \text { and } \llbracket \psi \rrbracket_{\mathcal{M}, u, w}=1 . \\
\text { f. } \llbracket \square \varphi \rrbracket_{\mathcal{M}, u, w}=1 \text { iff, for all } w^{\prime} \in W, \llbracket \varphi \rrbracket_{\mathcal{M}, u, w^{\prime}}=1 .
\end{array}
$$

By saying that a sentence $\varphi$ is true simpliciter in a model $\mathcal{M}$ and a world $u \in W$ iff $\llbracket \varphi \rrbracket_{\mathcal{M}, u, u}=1$, and that $\varphi$ is a priori true in a model iff it's true simpliciter in all worlds in the model, one can interpret the names and predicates in (1) and (2) to yield the desired result.

But two-dimensionalism isn't the only theory on the market that purports to account for the distinction between necessary and a priori truths. In this paper, I will look at two alternatives, one by Scott Soames (which I will call Metaphysically impossible worlds) and one by Kathrin Glüer-Pagin and Peter Pagin (which I will call Switcher semantics), and argue that both of them fail.

\section{Metaphysically impossible worlds}

On the face of it, the phenomenon of a posteriori necessities seems to suggest that there are epistemic or conceptual possibilities that aren't metaphysically possible. Or, in possible worlds parlance, that there are conceptually possible worlds that aren't metaphysically possible. In a standard possible worlds framework, the idea would then be to treat metaphysical possibility as some kind of restricted possibility, analogous to natural or nomic possibility (possibility according to the laws of nature). Metaphysical possibility would then be something like possibility according to the laws of metaphysics. The most liberal form of possibility is conceptual possibility. Indeed, this is what Lycan $(2009$, p. 78$)$ takes to be "the standard picture of logical space":

Consider the standard picture of logical space, featuring ever-larger concentric circles. We can start with the usual three grades of possibility, nomic, metaphysical, and conceptual; the nomically possible worlds are a proper subset of the metaphysically possible, which in turn are a proper subset of the conceptually possible. 
First of all, the standard picture of logical space has a general problem. Say that $\mathcal{M}=\langle W, D, R, I\rangle$ is a model of a language with a metaphysical necessity operator $\square$, where $W$ is a set of conceptually possible worlds, $D$ is a domain of objects, $R \subseteq W \times W$ is an accessibility relation and $I$ is an interpretation function. On the standard picture, there is a proper subset $U \subset W$ consisting of the metaphysically possible worlds such that

$$
\llbracket \square \varphi \rrbracket_{\mathcal{M}, w}=1 \text { iff, for all } w^{\prime} \in U, \llbracket \varphi \rrbracket_{\mathcal{M}, w^{\prime}}=1 .
$$

Assume, moreover, that

(7) $\varphi$ is a priori true in $\mathcal{M}$ iff $\varphi$ is true in all conceptually possible worlds in $\mathcal{M}$.

Arguably,

(8) It's a priori true that: if Mark Twain is Herman Melville, then necessarily, Mark Twain is Herman Melville.

but

(9) It's not a priori true that: Mark Twain isn't Herman Melville.

Granted as much, it follows that

(10) Necessarily, Mark Twain is Herman Melville.

using the classical interpretation of negation and implication. ${ }^{1}$ What this shows is that the standard picture, "featuring ever larger concentric circles", at least needs modification. Metaphysical possibility is better understood as a relation $R \subseteq W \times W$ between worlds than as a property $U \subseteq W$. Relative to each conceptually possible world, there is a set of metaphysically possible worlds. Indeed, the standard interpretation of the necessity operator in modal logic takes this form:

$$
\llbracket \square \varphi \rrbracket_{\mathcal{M}, w}=1 \text { iff, for all } w^{\prime} \in W \text { such that } w R w^{\prime}, \llbracket \varphi \rrbracket_{\mathcal{M}, w^{\prime}}=1 .
$$

But even on this modification, the standard picture is problematic. One prominent proponent, Scott Soames, writes (Soames 2006, p. 291):

Empirical evidence is required to rule out certain impossible, but nevertheless coherently conceivable and epistemologically relevant, world-states which (i) cannot be known a priori not to obtain, and (ii) are such that the necessary a posteriori truths are false with respect to those world-states. ${ }^{33}$

and adds in footnote 33 that

I here assume that names [...] rigidly designate the same thing with respect to all world-states, metaphysically possible or not.

\footnotetext{
${ }^{1}$ By (7) and (9), there's $w \in W$ such that $\llbracket$ Mark Twain is Herman Melville $\rrbracket_{\mathcal{M}, w}=1$. By (8), it follows that $\llbracket$ Necessarily, Mark Twain is Herman Melville $\rrbracket_{\mathcal{M}, w}=1$. By (6), we get $\llbracket$ Mark Twain is Herman Melville $\rrbracket_{\mathcal{M}, w^{\prime}}=1$ for all $w^{\prime} \in U$.
} 
According to this picture, while water is $\mathrm{H} 2 \mathrm{O}$ in all metaphysically possible worlds, there are conceptually possible worlds in which it's not. This is supposed to explain why (2) is a posteriori. I will return to the question of whether this is a reasonable move to make or not. Either way, the same strategy obviously won't work for identity claims like (1), at least not if names are treated as rigid designators. Soames bites the bullet by denying that (1) is a posteriori. In order to account for the fact that a perfectly rational and competent speaker nevertheless may fail to recognize its truth, he argues for the (in my view highly implausible) thesis that (Soames 2006, p. 279):

Sentences $\mathrm{S}_{1}$ and $\mathrm{S}_{2}$ may mean the same thing, and express the same proposition $\mathrm{p}$, even though a competent speaker who understands both sentences, and associates them with $\mathrm{p}$, does not realize that they express the same proposition.

As a case in point, we can let $\mathrm{S}_{1}$ be Mark Twain is Mark Twain, and let $\mathrm{S}_{2}$ be Mark Twain is Samuel Clemens.

Returning to (2), Soames accepts that this sentence is a posteriori. On his view, that means there is a conceptually possible but metaphysically impossible world in which an object is both a pure quantity of water and a pure quantity of XYZ. But, as Jackson (2010, p. 91) argues, the existence of such a world is actually hard to conceive of. For suppose there is one. Either the object in question is water, or it isn't. If it is, it's $\mathrm{H} 2 \mathrm{O}$. But being both $\mathrm{H} 2 \mathrm{O}$ and $\mathrm{XYZ}$ is conceptually impossible, just as being both $\mathrm{H} 2 \mathrm{O}$ and $\mathrm{C} 5 \mathrm{H} 5 \mathrm{OH}$ (ethanol) is conceptually impossible. It's conceptually impossible for a quantity to both contain carbon atoms and not contain carbon atoms. Therefore, contrary to our assumption, the world in which this is supposed to take place will be conceptually impossible. But if, on the other hand, the object in question isn't water, but only superficially like water, then the world in question isn't metaphysically impossible, again contrary to our assumption.

Presumably, this is why Soames (2007, p. 257) prefers to think of possible worlds as a maximally a priori consistent sets of Russellian propositions, rather than the other way around. Say that WATER is the property of being a pure quantity of water, $\mathrm{H} 2 \mathrm{O}$ is the property of being a pure quantity of $\mathrm{H} 2 \mathrm{O}$, and $X Y Z$ is the property of being a pure quantity of XYZ. It's perhaps easier to conceive of the existence of such a set containing both $\langle W A T E R, o\rangle$ and $\langle X Y Z, o\rangle$, than to accept the existence of a conceptually possible but metaphysically impossible world in which an object $o$ is both a pure quantity of water and a pure quantity of XYZ. However, if science is right (modulo some niceties concerning the actual chemical composition of water), the property of being a pure quantity of water is identical to the property of being a pure quantity of $\mathrm{H} 2 \mathrm{O}$, in which case $\langle W A T E R, o\rangle=\langle H 2 O, o\rangle$. Every set of Russellian propositions containing the former will then also contain the latter. But any set of Russellian propositions containing both $\langle H 2 O, o\rangle$ and $\langle X Y Z, o\rangle$ will (arguably) be a priori inconsistent, contrary to what Soames thinks. 


\section{Switcher semantics}

A different, but one-dimensional, explanation of the necessary a posteriori has been offered by Glüer and Pagin (2006, 2008, 2012). In order to explain why (1) is a posteriori, names have non-constant intensions. As in the case of the two-dimensional framework, this is formally consistent with any kind of meta-semantic story about reference determination (causal or descriptivist). Undeterred by Kripke's epistemic argument, however, the authors prefer to think of reference of a name as determined by some traditional cluster of descriptions. In order to explain the truth of (3), the $\square$ operator functions as an evaluation switcher, evaluating sentences containing names with respect to the referents the names have in the actual world, rather than the referents they have in the world of evaluation. Something similar happens in the case of predicates, which is supposed to explain why (4) is true and (2) is a posteriori.

I will now present a simplified version of their semantic theory. We will also ignore variables and quantifiers. The simplifications will not matter for my argument. A model $\mathcal{M}=\left\langle W, D, w^{*}, I\right\rangle$ has a set $W$ of possible worlds, a domain $D$ of objects and a distinguished member $w^{*} \in W$ (the actual world). The interpretation of a name $a$ is a function $I(a): W \rightarrow D$, and the interpretation of an $n$-place predicate $P$ is a pair $I(P)=\left\langle I_{m}(P), I_{u}(P)\right\rangle$ of functions $I_{m}(P): W \rightarrow \mathcal{P}\left(D^{n}\right)$ and $I_{u}(P): W \rightarrow$ $\mathcal{P}\left(D^{n}\right)$. The extension $I_{m}(=)(w)=I_{u}(=)(w)$ of the identity predicate is the identity relation on $D$ for all $w \in W$.

The idea is for $I_{m}(P)$ to be the manifest property (or relation) associated with $P$, while $I_{u}(P)$ is the underlying property (or relation) that realizes $I_{m}(P)$ in the actual world $w^{*}$. Since one and the same manifest property (being watery, for instance) may be realized by different underlying properties in other worlds (XYZ-worlds, for instance), it may be the case that $I_{m}(P)(w) \neq I_{u}(P)(w)$ when $w \neq w^{*}$. For certain predicates (the single-property associated ones), it will be the case that $I_{m}(P)=$ $I_{u}(P)$. But for others (the dual-property associated ones), it will not. Examples of single-property associated predicates in English are bachelor, unmarried, $\mathrm{H} 2 \mathrm{O}$ and the identity predicate. Examples of dual-property associated predicates are natural kind predicates such as water and tiger. In order to deal with certain Twin Earth scenarios, the authors have a context parameter in their semantics. Since it will not be relevant to my discussion, I have omitted it here.

Truth for sentences is defined by using two valuation functions, a possibilist function $\llbracket \cdot \rrbracket^{\mathrm{P}}$ and an actualist function $\llbracket \cdot \rrbracket^{\mathrm{A}}$, as follows:

$$
\begin{array}{ll}
\text { a. } & \llbracket a \rrbracket_{\mathcal{M}, w}^{\mathrm{P}}=I(a)(w) . \\
\text { b. } & \llbracket P \rrbracket_{\mathcal{M}, w}^{\mathrm{P}}=I_{m}(P)(w) . \\
\text { c. } & \llbracket P a_{1} \ldots a_{n} \rrbracket_{\mathcal{M}, w}^{\mathrm{P}}=1 \text { iff }\left\langle\llbracket t_{1} \rrbracket_{\mathcal{M}, w}^{\mathrm{P}}, \ldots, \llbracket t_{n} \rrbracket_{\mathcal{M}, w}^{\mathrm{P}}\right\rangle \in \llbracket P \rrbracket_{\mathcal{M}, w}^{\mathrm{P}} . \\
\text { d. } & \llbracket \neg \varphi \rrbracket_{\mathcal{M}, w}^{\mathrm{P}}=1 \text { iff } \llbracket \varphi \rrbracket_{\mathcal{M}, w}^{\mathrm{P}}=0 . \\
\text { e. } & \llbracket \varphi \wedge \psi \rrbracket_{\mathcal{M}, w}^{\mathrm{P}}=1 \text { iff } \llbracket \varphi \rrbracket_{\mathcal{M}, w}^{\mathrm{P}}=1 \text { and } \llbracket \psi \rrbracket_{\mathcal{M}, w}^{\mathrm{P}}=1 . \\
\text { f. } & \llbracket \square \varphi \rrbracket_{\mathcal{M}, w}^{\mathrm{P}}=1 \text { iff, for all } w^{\prime} \in W, \llbracket \varphi \rrbracket_{\mathcal{M}, w^{\prime}}^{\mathrm{A}}=1 . \\
\text { a. } & \llbracket a \rrbracket_{\mathcal{M}, w}^{\mathrm{A}}=I(a)\left(w^{*}\right) . \\
\text { b. } & \llbracket P \rrbracket_{\mathcal{M}, w}^{\mathrm{A}}=I_{u}(P)(w) .
\end{array}
$$



c. $\llbracket P a_{1} \ldots a_{n} \rrbracket_{\mathcal{M}, w}^{\mathrm{A}}=1$ iff $\left\langle\llbracket t_{1} \rrbracket_{\mathcal{M}, w}^{\mathrm{A}}, \ldots, \llbracket t_{n} \rrbracket_{\mathcal{M}, w}^{\mathrm{A}}\right\rangle \in \llbracket P \rrbracket_{\mathcal{M}, w}^{\mathrm{A}}$.
d. $\llbracket \neg \varphi \rrbracket_{\mathcal{M}, w}^{\mathrm{A}}=1$ iff $\llbracket \varphi \rrbracket_{\mathcal{M}, w}^{\mathrm{A}}=0$.
e. $\llbracket \varphi \wedge \psi \rrbracket_{\mathcal{M}, w}^{\mathrm{A}}=1$ iff $\llbracket \varphi \rrbracket_{\mathcal{M}, w}^{\mathrm{A}}=1$ and $\llbracket \psi \rrbracket_{\mathcal{M}, w}^{\mathrm{A}}=1$.
f. $\llbracket \square \varphi \rrbracket_{\mathcal{M}, w}^{\mathrm{A}}=1$ iff, for all $w^{\prime} \in W, \llbracket \varphi \rrbracket_{\mathcal{M}, w^{\prime}}^{\mathrm{A}}=1$.

A sentence $\varphi$ is true in the actual world iff $\llbracket \varphi \rrbracket_{\mathcal{M}, w^{*}}^{\mathrm{P}}=1$. For each sentence $\varphi$, let $[\varphi]^{\mathrm{P}}=\left\{w \in W: \llbracket \varphi \rrbracket_{\mathcal{M}, w}^{\mathrm{P}}=1\right\}$ and $[\varphi]^{\mathrm{A}}=\left\{w \in W: \llbracket \varphi \rrbracket_{\mathcal{M}, w}^{\mathrm{A}}=1\right\}$ be its possibilist and acutalist intension, respectively. We say that a sentence $\varphi$ is contingent iff $\emptyset \neq[\varphi]^{P} \neq W$, it's necessary iff $[\varphi]^{P}=W$, and its necessitation is true iff $\square \varphi$ is true in the actual world. All these notions are of course relative to a model.

Now, assuming that Mark Twain $(t)$ is Samuel Clemens $(c)$, and provided that $\mathcal{M}$ is the intended interpretation, it will indeed be the case that

$$
\llbracket t=c \rrbracket_{\mathcal{M}, w^{*}}^{\mathrm{P}}=1 .
$$

and

$$
\llbracket \square t=c \rrbracket_{\mathcal{M}, w^{*}}^{\mathrm{P}}=1 .
$$

Moreover, as long as there is a world $w \in W$ such that $I(t)(w) \neq I(c)(w)$, we have

$$
\llbracket t=c \rrbracket_{\mathcal{M}, w}^{\mathrm{P}}=0 .
$$

which means that (1) is contingent. This is also supposed to explain why it's a posteriori. Glüer and Pagin (2012, p. 177) hypothesizes that, on the assumption that a sentence $\varphi$ is true (in the actual world), its modal and epistemic status will be determined by the so-called NN-pattern, in their terminology:

\begin{tabular}{lll}
$\varphi$ & necessary & necessitation \\
\hline a priori necessary & yes & true \\
a posteriori necessary & no & true \\
a priori contingent & yes & false \\
a posteriori contingent & no & false
\end{tabular}

from which it follows that

$$
\varphi \text { is a priori true iff } \varphi \text { is necessary. }
$$

Arguably, however, this hypothesis is false. The sentence

$$
\square t=c
$$

will be necessary according to the semantics (although it's intuitively not a priori), and

$$
\square t=c \rightarrow t=c
$$

will not be necessary according to the semantics (although it's intuitively a priori). Both sentences violate the NN-pattern, and they belong to the fragment for which the semantics is defined. 
Moreover, assuming that the hypothesis (18) is correct, the set of a priori truths will not be closed under what the authors take to be the appropriate notion of logical consequence (real-world consequence ${ }^{2}$ ), since (20) is true in the actual world of all models (it's real-world valid). The authors seem to be aware of this problem, however. As they point out,

$$
a=b \rightarrow \square a=b
$$

will be real-world valid, and yet contingent on their semantics.

It's therefore not clear how apriority is to be defined relative to $\mathcal{M}$. I will now look at three alternatives, and argue that none of them can succeed. Obviously, this is no proof that apriority cannot be defined within the framework of switcher semantics. If such a claim were to be proven at all, it would first have to be made precise. I shall not attempt to provide such a precisification here. I will merely show that the notion in question is hard to define in terms of the possibilist and actualist intensions of the expressions involved.

\subsection{By closure}

Perhaps the scope of (18) should be limited to non-modal sentences, and the set of a priori truths thus defined extended to modal sentences by closure under real-world consequence? Then (20) would come out as a priori. But the suggestion will not work for the following reason. Even if the sentence

$$
\operatorname{Bachelor}(a) \rightarrow \operatorname{Unmarried}(a)
$$

is included in the set of a priori non-modal truths, its necessitation (which, intuitively, is also a priori)

$$
\square(\operatorname{Bachelor}(a) \rightarrow \operatorname{Unmarried}(a))
$$

is not a real-world consequence of it. To see why, suppose $\mathcal{M}=\left\langle W, D, w^{*}, I\right\rangle$ is the intended interpretation of our language, and let $\mathcal{M}^{\prime}=\left\langle W, D, w^{*}, I^{\prime}\right\rangle$ be just like $\mathcal{M}$ except that, for some $w \neq w^{*}, I^{\prime}(a)\left(w^{*}\right) \in I_{u}^{\prime}$ (Bachelor) $(w)$ and $I^{\prime}(a)\left(w^{*}\right) \notin$ $I_{u}^{\prime}($ Unmarried $)(w)$. Clearly, all the non-modal a priori truths relative to $\mathcal{M}$ will be true in the actual world of $\mathcal{M}^{\prime}$, but (23) will be false.

\subsection{By induction}

Perhaps one could say that (19) is a posteriori because its immediate constituent is contingent, and that (23) is a priori because its immediate constituent is necessary? Perhaps, but a different explanation would then be needed for $\square \square t=c$, which is also intuitively a posteriori although its immediate constituent is necessary, and for (20),

\footnotetext{
${ }^{2} \varphi$ is a real-world consequence of $\Gamma$ iff there's no model $\mathcal{M}=\left\langle W, D, w^{*}, I\right\rangle$ such that $\llbracket \psi \rrbracket \mathcal{M}, w^{*}=1$ for all $\psi \in \Gamma$ but $\llbracket \varphi \rrbracket_{\mathcal{M}, w^{*}}=0$. Likewise, $\varphi$ is real-world valid iff it's a real world consequence of the empty set of premises. Cf. Glüer and Pagin (2006, pp. 517-518). Observe that if a set of sentences is closed under real-world consequence, it will contain every real-world valid sentence.
} 
which is intuitively a priori although one of its immediate constituents is contingent. Perhaps one could instead say that (19) is a posteriori because the embedded sentence is a posteriori? Perhaps, but it's not clear how that would help us deal with (20). In general, it's impossible to determine the a priori status of a sentence in switcher semantics by looking at the a priori status of its immediate constituents and their intensions. To see why, let $t$ be the name Mark Twain and $c$ be the name Samuel Clemens. Relative to some model $\mathcal{M}=\left\langle W, D, w^{*}, I\right\rangle$, such that $I(t)\left(w^{*}\right)=I(c)\left(w^{*}\right)$, suppose we want to define the characteristic functions $A T$ and $A F$ of the set of a priori truths and falsehoods in an intuitively satisfying manner, and that we want to do it inductively over the complexity of sentences using their possibilist and actualist intensions. Presumably, the base clauses of such a definition would be
a. $A T\left(P a_{1}, \ldots, a_{n}\right)=1$ iff $\left[P a_{1} \ldots a_{n}\right]^{\mathrm{P}}=W$
b. $\quad A F\left(P a_{1}, \ldots, a_{n}\right)=1$ iff $\left[P a_{1} \ldots a_{n}\right]^{\mathrm{P}}=\varnothing$

Also, the inductive clauses for negation would presumably be
a. $A T(\neg \varphi)=A F(\varphi)$
b. $A F(\neg \varphi)=A T(\varphi)$

The precise nature of these clauses will not matter for my argument, and are given for the purpose of illustration only. As for the operators $\vee$ and $\square$ (the other operators are assumed to be defined in terms of them), we assume that there are functions $T_{\vee}, F_{\vee}, T_{\square}, F_{\square}$ such that

$$
\begin{aligned}
\text { a. } & A T(\varphi \vee \psi)= \\
& T_{\vee}\left(A T(\varphi), A T(\psi), A F(\varphi), A F(\psi),[\varphi]^{\mathrm{P}},[\psi]^{\mathrm{P}},[\varphi]^{\mathrm{A}},[\psi]^{\mathrm{A}}\right) \\
\text { b. } & A F(\varphi \vee \psi)= \\
& F_{\vee}\left(A T(\varphi), A T(\psi), A F(\varphi), A F(\psi),[\varphi]^{\mathrm{P}},[\psi]^{\mathrm{P}},[\varphi]^{\mathrm{A}},[\psi]^{\mathrm{A}}\right)
\end{aligned}
$$

and

$$
\begin{aligned}
& \text { a. } \quad A T(\square \varphi)=T_{\square}\left(A T(\varphi), A F(\varphi),[\varphi]^{\mathrm{P}},[\varphi]^{\mathrm{A}}\right) \\
& \text { b. } \quad A F(\square \varphi)=F_{\square}\left(A T(\varphi), A F(\varphi),[\varphi]^{\mathrm{P}},[\varphi]^{\mathrm{A}}\right)
\end{aligned}
$$

respectively. This is tantamount to assuming that the a priori status of a sentence can be determined by the a priori status of its immediate constituents and their possibilist and acutalist intensions. We will now show that this cannot be done in an intuitively satisfying manner. To this end, let $T$ be the intuitively a priori true sentence $\square t=t$, and let $C$ be the intuitively a posteriori true sentence $\square t=c$. Intuitively, we thus want it to be the case that

$$
\begin{array}{ll}
\text { a. } & A T(T)=1 \\
\text { b. } & A T(C)=0
\end{array}
$$

Now, let $P$ be some intuitively a posteriori sentence unrelated to $T$ and $C$, e.g. It's raining. Since every sentence $\varphi$ is equivalent to $(\varphi \wedge P) \vee(\varphi \wedge \neg P)$ in propositional logic, we want it intuitively to be the case that 

a. $A T((T \wedge P) \vee(T \wedge \neg P))=A T(T)$
b. $A T((C \wedge P) \vee(C \wedge \neg P))=A T(C)$

Moreover, since $P$ is neither a priori true nor a priori false, we also want it to be the case that

$$
\begin{aligned}
& A T(T \wedge P)=A T(T \wedge \neg P)=A F(T \wedge P)=A F(T \wedge \neg P)= \\
& =A T(C \wedge P)=A T(C \wedge \neg P)=A F(C \wedge P)=A F(C \wedge \neg P)=0
\end{aligned}
$$

According to switcher semantics, $T$ and $C$ have the same intensions, i.e. $[T]^{\mathrm{P}}=[C]^{\mathrm{P}}$ and $[T]^{\mathrm{A}}=[C]^{\mathrm{A}}$. It follows that
a. $[T \wedge P]^{\mathrm{P}}=[C \wedge P]^{\mathrm{P}}$
b. $[T \wedge P]^{\mathrm{A}}=[C \wedge P]^{\mathrm{A}}$
c. $[T \wedge \neg P]^{\mathrm{P}}=[C \wedge \neg P]^{\mathrm{P}}$
d. $[T \wedge \neg P]^{\mathrm{A}}=[C \wedge \neg P]^{\mathrm{A}}$

By (26-a), (30) and (31), we then get

$$
A T((T \wedge P) \vee(T \wedge \neg P))=A T((C \wedge P) \vee(C \wedge \neg P))
$$

contradicting (28) and (29).

This shows that we cannot determine whether a sentence is a priori by looking only at its constituents one level down in the construction tree with respect to the values of $A T, A F$ and their intensions $[\cdot]^{\mathrm{P}}$ and $[\cdot]^{\mathrm{A}}$. This generalizes: there is no finite number $n$ such that it always suffices to look $n$ level down. To see why, define the unary operator $\bigwedge^{i}$ recursively:

$$
\begin{aligned}
& \text { a. } \bigwedge^{0} \varphi=\varphi \wedge \varphi . \\
& \text { b. } \bigwedge^{i+1} \varphi=\left(\bigwedge^{i} \varphi\right) \wedge\left(\bigwedge^{i} \varphi\right) .
\end{aligned}
$$

Assuming that $A T$ and $A F$ are invariant under propositional logical equivalence, we intuitively want it to be the case that

$$
A T\left(\bigwedge^{n}(T \wedge P) \vee \bigwedge^{n}(T \wedge \neg P)\right)=1 .
$$

and

$$
A T\left(\bigwedge^{n}(C \wedge P) \vee \bigwedge^{n}(C \wedge \neg P)\right)=0 .
$$

However, at each level $1 \leq k \leq n$ in the construction tree, the constituents of the sentences in (34) and (35) will be identical with respect to the values of $A T, A F,[\cdot]^{\mathrm{P}}$ and $[\cdot]^{\mathrm{A}}$, and the tree structures will be identical down to level $n$.

\subsection{By variance}

As a last resort, one could of course suggest the following. Suppose $\mathcal{M}=$ $\left\langle W, D, w^{*}, I\right\rangle$ is the intended interpretation of our language. Define the class of models 


$$
[\mathcal{M}] \approx=\{\langle W, D, w, I\rangle: w \in W\} .
$$

The reader may observe that $[\mathcal{M}] \approx=\left\{\mathcal{M}^{\prime}: \mathcal{M} \approx \mathcal{M}^{\prime}\right\}$, where $\approx$ is the relation of variance introduced by Davies and Humberstone (1980, p. 2). ${ }^{3}$ One could then perhaps say that

$\varphi$ is a priori true (false) iff $\varphi$ is true (false) in the actual world of $\mathcal{M}^{\prime}$ for all $\mathcal{M}^{\prime} \in[\mathcal{M}] \approx$.

That would indeed make (19) a posteriori, and make (20) a priori. But it would still make

$$
\square(\operatorname{Water}(a) \rightarrow \mathrm{H} 2 \mathrm{O}(a))
$$

a priori, the reason being that $I_{u}$ (Water) $=I_{u}(\mathrm{H} 2 \mathrm{O})$ for all $\mathcal{M}^{\prime} \in[\mathcal{M}] \approx$. This highlights the fact that, in order to make the analytic/synthetic distinction for sentences involving natural kind predicates, it seems one needs to specify a two-dimensional intension for each such predicate. It doesn't seem enough to specify two onedimensional intensions for each of them, as in the case of switcher semantics.

A more general problem with the variance approach, however, is that it looks a lot like two-dimensionalism in disguise. Glüer and Pagin (2006, p. 530) take it to be a crucial difference between switcher semantics and two-dimensionalism that a sentence like

The teacher of Alexander is the actual teacher of Alexander.

will be a priori in two-dimensional semantics, but not in switcher semantics (on the standard interpretation of the actuality operator). But on the present definition of apriority, this difference vanishes. (39) will still be contingent in the technical sense, but the notion of contingency will have lost its explanatory role. As we saw in the previous section, it's hard to see how this notion can play a role in determining the a priori status of sentences in switcher semantics.

\section{Conclusion}

Postulating the existence of conceptually possible but metaphysically impossible worlds doesn't seem to help in accounting for the phenomenon of a posteriori necessities, especially not if names are treated as rigid designators. Either way, some of the conceptually possible worlds needed are hard to conceive of on closer inspection. As for switcher semantics, the original proposal doesn't work for certain modal sentences, and I have shown that it cannot be easily amended. The lesson, I take it, is that it's hard to explain the phenomenon of a posteriori necessities by merely assigning two one-dimensional intensions to each expression. At least in the case of natural kind predicates, one seems to need the whole two-dimensional matrix.

\footnotetext{
3 The relation is defined as follows: for any models $\mathcal{M}_{1}=\left\langle W_{1}, D_{1}, w_{1}^{*}, I_{1}\right\rangle$ and $\mathcal{M}_{2}=\left\langle W_{2}, D_{2}, w_{2}^{*}, I_{2}\right\rangle$ with $w_{1}^{*} \in W_{1}$ and $w_{2}^{*} \in W_{2}$, we have $\mathcal{M}_{1} \approx \mathcal{M}_{2}$ just in case $W_{1}=W_{2}, D_{1}=D_{2}$ and $I_{1}=I_{2}$. 
Acknowledgements Open access funding provided by Stockholm University.

Open Access This article is distributed under the terms of the Creative Commons Attribution 4.0 International License (http://creativecommons.org/licenses/by/4.0/), which permits unrestricted use, distribution, and reproduction in any medium, provided you give appropriate credit to the original author(s) and the source, provide a link to the Creative Commons license, and indicate if changes were made.

\section{References}

Davies, M., \& Humberstone, L. (1980). Two notions of necessity. Philosophical Studies, 38(1), 1-31.

Glüer, K., \& Pagin, P. (2006). Proper names and relational modality. Linguistics and Philosophy, 29(5), 507-535.

Glüer, K., \& Pagin, P. (2008). Relational modality. Journal of Logic, Language and Information, 17(3), $307-322$.

Glüer, K., \& Pagin, P. (2012). General terms and relational modality. Noûs, 46(1), 159-199.

Jackson, F. (2010). Language, names, and information. Oxford: Wiley-Blackwell.

Lycan, W. G. (2009). Serious metaphysics: Frank Jackson's defense of conceptual analysis. In I. Ravenscroft (Ed.), Minds, ethics, and conditionals: Themes from the philosophy of Frank Jackson. Oxford: Oxford University Press.

Soames, S. (2006). Kripke, the necessary a posteriori, and the two-dimensionalist heresy. In GarciaCarpintero (Ed.), Two-dimensional semantics (pp. 272-292). Oxford: Clarendon Press.

Soames, S. (2007). Actually. Aristotelian Society Supplementary, 81(1), 251-277.

Publisher's Note Springer Nature remains neutral with regard to jurisdictional claims in published maps and institutional affiliations. 